\title{
Is orchiectomy always necessary in retroperitoneal extragonadal germ cell tumours?
}

\author{
Robert J. Hamilton, MD, MPH, FRCSC \\ Princess Margaret Cancer Centre, University Health Network Assistant Professor, Dept. of Surgery (Urology), University of Toronto
}

See related article on page 381.

Cite as: Can Urol Assoc J 2015;9 (11-12):385-6. hitp://dx.doi.org/10.5489/cuai.3506 Published online December 14, 2015.

$P$ unjani and colleagues review their 35-year, single-centre experience of 14 patients treated with chemotherapy for retroperitoneal extragonadal germ cell tumours (GCT) with no ultrasonographic or palpable abnormality in the testicles. Eight underwent orchiectomy at the time of retroperitoneal lymph node dissection (RPLND), two (14\%) had viable disease, and one had intratubular germ cell neoplasia (ITGCN). This paper raises some important points.

The authors, while not formally recommending postchemotherapy orchiectomy in all patients, hint that retroperitoneal GCTs may, in fact, all be testicular GCTs and we should carefully evaluate the need for orchiectomy in these patients.

I feel we should be cautious advocating for orchiectomy for all based on the limited series that have examined this. At Princess Margaret, we agree with orchiectomy when suspicion of a lesion is present in the testicle after chemotherapy. In the few case series that have examined this, pathology in the testicle post-chemotherapy harbours the same risk of viable GCT or teratoma as residual masses elsewhere in the body and, thus, orchiectomy appears warranted. ${ }^{1}$ However, when there is no clinical or radiographic abnormality in the testicle preand post-chemotherapy, such as the scenario described in this study, we typically survey the testicle and avoid orchiectomy.

The risk of this practice is subsequent appearance of an ipsilateral metachronous testicular GCT - whether from growth of a chemo-resistant GCT or progression of ITGCN to GCT. Metachronous tumour development in this setting has been estimated at $10 \%$ over 10 years. ${ }^{2}$ The risk is higher when the retroperitoneum contains nonseminoma (14\%) as compared to seminoma (1.4\%). However, the majority $(70 \%)$ of metachronous testicular tumours are seminomas and the stage distribution and clinical behaviour mirrors new testicular cancers as opposed to an aggressive metastatic counterpart; thus, most can be managed with orchiectomy alone followed by surveillance.

This pattern is homologous to the risk of subsequent development of a contralateral testicular GCT in a patient with a prior testicular GCT. This risk is as high as $5 \%,{ }^{3}$ and the tumours are typically low-stage seminomas that can be managed with orchiectomy alone. ${ }^{3}$ The similarity between metachronous ipsilateral tumour in extragonadal GCT and contralateral second testicular primary is because both are believed due to the presence of ITGCN. Testicular biopsies at the time of diagnosis of retroperitoneal GCT suggest ITGCN in $20-40 \%,{ }^{4}$ and it is believed that one-third of ITGCN present will persist in a testicle after chemotherapy, with half of ITGCN subsequently developing into a GCT.

The consequence of performing orchiectomy in all patients with retroperitoneal GCT and no testicular abnormality is the risk of removing a normal testicle in up to $90 \%$. This has minor surgical morbidity but potentially greater impact on fertility and gonadal hormonal function, which are not inconsequential in men treated with chemotherapy. ${ }^{5}$ The normal-appearing testicle can be followed sonographically and orchiectomy performed at first sign of abnormality.

The data presented by Punjani et al. highlight the need for large, multi-institution registry data to capture retroperitoneal primary GCTs treated with chemotherapy. One disadvantage of single-centre series is the need to go back several decades to have enough patients to analyze. However, modern-era chemotherapy is more effective in eradicating viable GCT in the retroperitoneum and thus probably also the testicle. ${ }^{6}$ Thus ideally a large registry series will have sufficient numbers of modern-era patients and we may learn the metachronous rate is even lower than $10 \%$. However, until such data are available, we continue to feel surveillance of the sonographically normal testicle post-chemotherapy is appropriate.

Competing interests: Dr. Hamilton is a member of Advisory Boards with Abbvie, Janssen, Astellas, and Bayer. He has also participated in clinical trials with Janssen. 
Hamilton

\section{References}

1. Reddy BV, Sivakanth A, Naveen Babu G, et al. Role of chemotherapy prior to orchiectomy in metastatic testicular cancer-is testis really a sanctuary site? Ecancermedicalscience 2014;8:407.

2. Hartmann JT, Fosså SD, Nichols CR, et al. Incidence of metachronous testicular cancer in patients with extragonadal germ cell tumors. J Natl Cancer Inst 2001;93(22):1733-8. http://dx.doi.org/10.1093/ inci/93.22.1733

3. Fosså SD, Chen J, Schonfeld SJ, et al. Risk of contralateral testicular cancer: A population-based study of 29515 U.S. men. J Natl Cancer Inst 2005;97(14):1056-66. http://dx.doi.org/10.1093/inci/dii185
4. Daugaard $\mathrm{G}$, Rorth $M$, Maase von der $\mathrm{H}$, et al. Management of extragonadal germ-cell tumors and the significance of bilateral testicular biopsies. Ann Oncol 1992;3(4):283-9.

5. Haugnes $\mathrm{HS}$, Bosl $\mathrm{GJ}$, Boer $\mathrm{H}$, et al. Long-term and late effects of germ cell testicular cancer treatment and implications for followup. J Clin Oncol 2012; 30(30):3752-63. http://dx.doi.org/10.1200/ JC0.2012.43.4431

6. Carver $B S$, Serio $A M$, Bajorin $D$, et al. Improved clinical outcome in recent years for men with metastatic nonseminomatous germ cell tumors. J Clin Oncol 2007;25(35):5603-8. http://dx.doi.org/10.1200/ JC0.2007.13.6283 